\title{
A Case of Ocular Angiostrongyliasis with Molecular Identification of the Species in Vietnam
}

\author{
Nguyen Van De ${ }^{1, *}$, Le Van Duyet ${ }^{2}$, Jong-Yil Chai ${ }^{3}$ \\ 'Department of Parasitology, Hanoi Medical University, Hanoi, Vietnam; 'Department of Immunology, Institute of Bio-Technology, Hanoi, Vietnam; \\ ${ }^{3}$ Department of Parasitology and Tropical Medicine, Seoul National University College of Medicine, Seoul 03080, Korea
}

\begin{abstract}
A 23-year-old female residing in a village of Cao Bang Province, North Vietnam, visited the Hospital of Hanoi Medical University in July 2013. She felt dim eyes and a bulge-sticking pain in her left eye for some days before visiting the hospital. In the hospital, a clinical examination, an eye endoscopy, and an operation were carried out. A nematode specimen was collected from the eye of this patient. The body of this worm was thin and long and measured $22.0 \times 0.3$ $\mathrm{mm}$. It was morphologically suggested as an immature female worm of Angiostrongylus cantonensis. By a molecular method using 18S rRNA gene, this nematode was confirmed as $A$. cantonensis. This is the first molecular study for identification of $A$. cantonensis in Vietnam.
\end{abstract}

Key words: Angiostrongylus cantonensis, eosinophilic meningoencephalitis, eye

\section{INTRODUCTION}

In the genus Angiostrongylus, a total of 21 species have been described, including 2 species which cause diseases in humans such as Angiostrongylus cantonensis and Angiostrongylus costaricensis $[1,2]$. A. cantonensis causes eosinophilic meningoencephalitis in humans damaging the brain tissues, which result in bleeding, inflammation, and granuloma formation [1]. Neurological symptoms vary with the region affected. On the other hand, A. costaricensis causes abdominal infections [2].

A. cantonensis was first found from the lungs of rats Rattus norvegicus and Rattus rattus in 1935 by Chen in China [2]. The first human case infected with $A$. cantonensis was reported by Nomura and Lin in 1945 in Taiwan [2]. In 1962, Rosen et al. [3] reported 2 cases of eosinophilic meningoencephalitis caused by A. cantonensis and called the worm as the rat lungworm. After then, A. cantonensis has been detected in Madagascar, Hawaii, South East Asia, and Japan [2]. Meanwhile, A. costaricensis was detected for the first time in Costa Rica, and then in Middle America and North America [2].

- Received 13 July 2015, revised 2 September 2015, accepted 29 September 2015.

*Corresponding author (ngvdeyhn@gmail.com)

(c) 2015, Korean Society for Parasitology and Tropical Medicine

This is an Open Access article distributed under the terms of the Creative Commons Attribution Non-Commercial License (http://creativecommons.org/licenses/by-nc/3.0) which permits unrestricted non-commercial use, distribution, and reproduction in any

medium, provided the original work is properly cited.
In Vietnam, eosinophilic meningoencephalitis caused by $A$. cantonensis was first detected in 1976 [4]. Phan [4] reported 2 human cases (3- and 7-year old) with symptoms like fever, vomiting, snatched legs, leg pain, paralyses, positive meningoencephalitis, and eosinophillia (29-59\%). In addition, An [5] reported 15 children cases (1-15 years old) of eosinophilic meningoencephalitis due to A. cantonensis infection in the National Children Hospital during 1995-2000. After then, De et al. [6,7] reported 5 cases of angiostrongyliasis in 2004 and another case in 2008. We report here an additional case of human angiostrongyliasis caused by A. cantonensis; the species was molecularly confirmed for the first time in Vietnam.

\section{CASE RECORD}

The patient was a 23-year-old female residing in the Chi Thao Village, Quang Yen District, Cao Bang Province, North Vietnam. In July 2013, she felt dim eyes and a bulge-sticking pain in her left eye for some days at home. She visited the provincial hospital and then the Hospital of Hanoi Medical University, Hanoi, Vietnam. In the hospital, a clinical examination and an eye endoscopy were carried out, and a nematode worm was seen in the left eye. This nematode specimen was collected from the eye of this patient by operation. The body of this worm was thin and long and measured $22 \mathrm{~mm}$ in length and $0.3 \mathrm{~mm}$ in width (Fig. 1). It was morphologically suggested as 


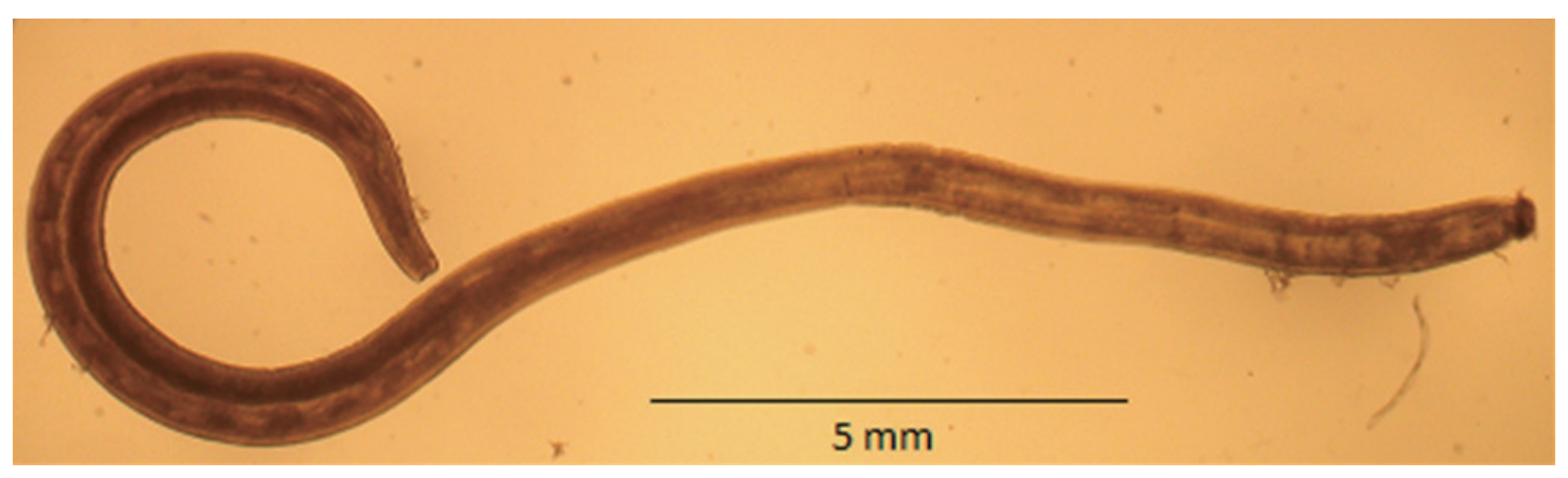

Fig. 1. An immature female worm of Angiostrongylus cantonensis collected from the human eye in this study.

Table 1. Sequencing of a portion of $18 \mathrm{~S}$ ribosomal RNA of different Angiostrongylus isolates from GenBank compared with Angiostrongylus cantonensis in Vietnam

\begin{tabular}{lcccccc}
\hline Notation & Origin & Host & Length & Species & GenBank no. & Author \\
\hline AC-VN & Vietnam & Human & 522 & A. cantonensis & - & This study \\
AC-NO & USA & Snail (Pomacea insularum) & 522 & A. cantonensis & GU587760 & Qvarnstrom et al. [12] \\
AC-BR & Brazil & Mollusk & 522 & A. cantonensis & GU733321 & Qvarnstrom et al. [12] \\
AC-SZ1 & China & Rat & 522 & A. cantonensis & GU540546 & Liu et al. [13] \\
AC-SZ2 & China & Rat & 522 & A. cantonensis & GU540547 & Liu et al. [13] \\
AC-QY & China & Rat & 522 & A. cantonensis & GU540549 & Liu et al. [13] \\
AC-WZ & China & Rat & 522 & A. cantonensis & GU540551 & Liu et al. [13] \\
AC-CR & Costa Rica & Mollusk & 522 & A. costaricensis & GU587745 & Qvarnstrom et al. [12] \\
AC-UK & UK & Vulpes & 522 & A. vasorum & GU045374 & Jefferies et al. [14] \\
\hline
\end{tabular}

an immature (female) worm of $A$. cantonensis.

By the molecular method using a portion of $18 S$ rRNA gene, this Vietnamese Angiostrongylus was sequenced and compared with different worldwide A. cantonensis isolates from GenBank (Table 1). A comparison was made on 522 nucleotides of a portion of $18 S$ rRNA gene between the Vietnamese Angiostrongylus and isolates from USA (AC-NO), Brazil (AC-BR), China (AC-SZ1, AC-SZ2, AC-QY, and AC-WZ), Costa Rica (AC-CR), and UK (AV-UK). The results revealed 99\% identity with other A. cantonensis reported in GenBank (Fig. 2).

\section{DISCUSSION}

The symptoms in angiostrongyliasis patients in Vietnam included headache (15/15), fever (12/15), vomiting (11/15), harden neck (8/15), and eosinophillia (31.5-48.5\%) [4]. In 2004, De et al. [6] also reported 5 cases (19-month to 10-yearold) of angiostrongyliasis with the symptoms of headache (5/5), fever (5/5), vomiting (4/5), snatched leg (5/5), disordered reflected knee (5/5), leg paralysis (5/5), positive meningoencephalitis (5/5), eosinophillia (5/5 with $19-30 \%)$, and positive ELISA with Angiostrongylus antigen (5/5). A worm was collected from 1 of the 5 patients [6]. These symptoms were similar to those reported previously in Taiwan [8]. However, eosinophilic meningoencephalitis due to A. cantonensis occurred more and more often in Vietnam [7]. The parasitic place of Angiostrongylus was usually the brain, but can be in other places, including the eyes. In 2001, Xuan et al. [9] also reported a case infected with $A$. cantonensis in the eye.

The incidence of ocular angiostrongyliasis is low; however, it can cause hypopsia or even vision loss, which would seriously affect the quality of the life of patients [10]. Feng et al. [10] reviewed total 42 ocular cases reported worldwide; nearly a half of them were from Thailand and the rest were from other Asian countries, including Sri Lanka, India, Taiwan, Vietnam, Malaysia, Japan, and Indonesia. In most cases, worms were found from the anterior chamber or vitreous fluid, and in a few cases they were from the retina or subretina [10]. The majority of the ocular cases was not associated with cerebrospinal symptoms; only 12 of the 42 cases suffered from eosinophilic meningitis [10]. In our case, the patient felt dim eyes and a bulge-sticking pain in her left eye; however, she developed no further symp- 

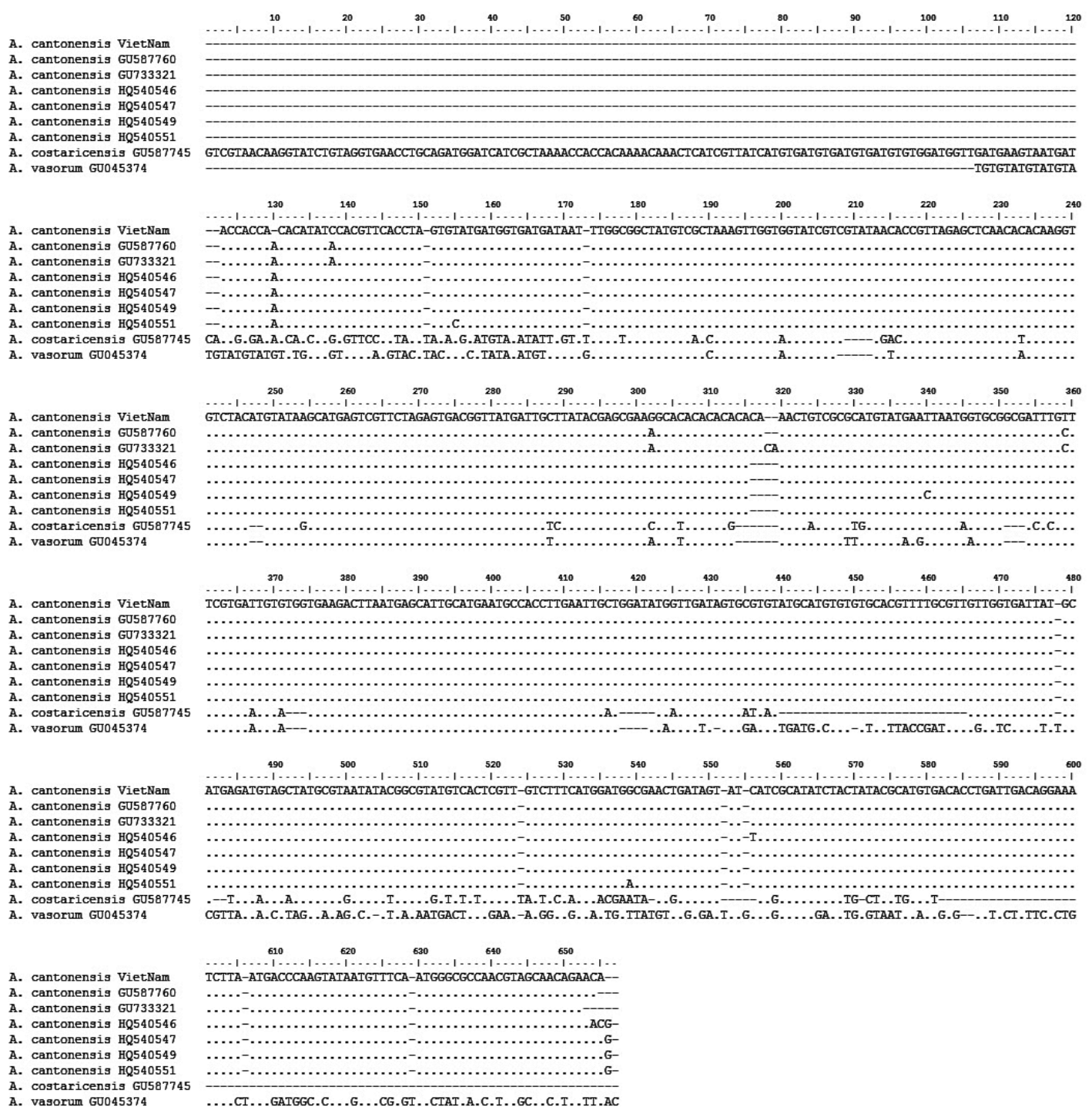

Fig. 2. Comparison of 522 nucleotides of a portion of $18 \mathrm{~S}$ ribosomal RNA gene (18S rRNA) between the Vietnamese Angiostrongylus cantonensis and isolates from USA (AC-NO), Brazil (AC-BR), China (AC-SZ1, AC-SZ2, AC-QY, AC-WZ), Costa Rica (AC-CR), and UK (AV-UK). Note similarities between the Vietnamese (A. cantonensis Vietnam) and other isolates. Mark (.) is similar to each other in nucleotides; mark (-) is mismatching.

toms like meningoencephalitis. After the surgical removal of the worm, her eye symptoms completely disappeared.

The phylogenetic tree of A. cantonensis Vietnam and other strains from different countries targeting a part of $18 \mathrm{~S} r \mathrm{RNA}$ nucleotide sequences was constructed by the neighbor-joining (NI) method using MEGA4.0 [11]. It has been shown that the Vietnamese A. cantonensis is located in the same group with other A. cantonensis isolates available in GenBank (Fig. 3). The present study is the first to identify the species of the nematode by a molecular method in Vietnam.

\section{ACKNOWLEDGMENTS}

This research was funded by Vietnam National Foundation for Science and Technology Development (NAFOSTED) under grant no. 106-YS.05-2014.08, and collaborated with the National Hospital of Eyes, Hanoi, Vietnam.

\section{CONFLICT OF INTEREST}

We have no conflict of interest related to this work. 


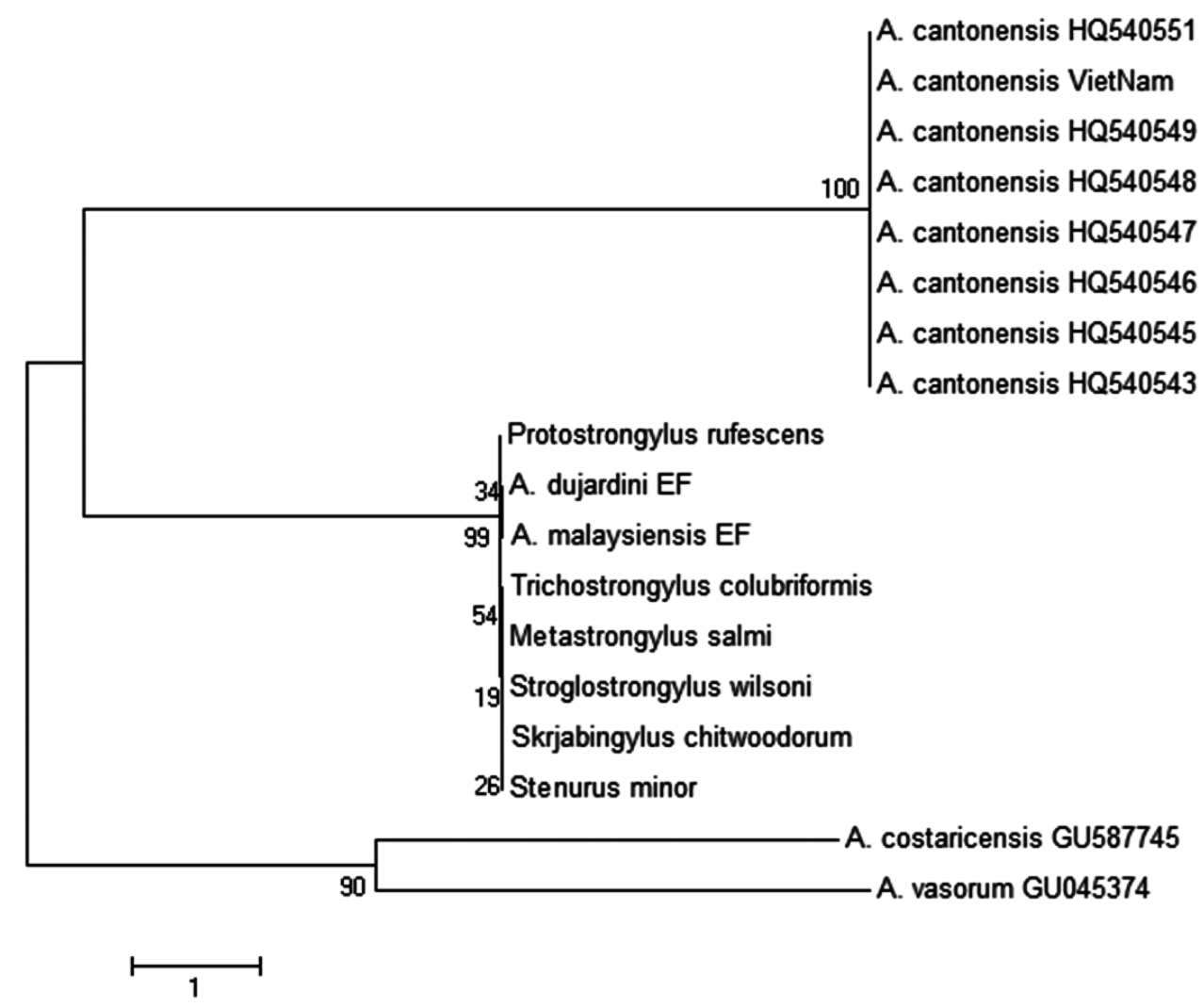

Fig. 3. Phylogenetic tree of Angiostrongylus cantonensis Vietnam and other isolates targeting a part of 18S rRNA nucleotide sequences estimated by the neighbor-joining (NJ) using MEGA4.0 [9]. Note: $A$. cantonensis Vietnam = Vietnamese Angiostrongylus; $A$. cantonensis - GenBank no. HQ540548; A. cantonensis - GenBank no. HQ540547; A. cantonensis - GenBank no. HQ540546; A. cantonensis GenBank no. HQ540545; A. cantonensis - GenBank no. HQ540543; A. cantonensis - GenBank no. HQ540551; A. cantonensis GenBank no. HQ540549; A. dujardini EF - GenBank no. EF514915; A. malaysiensis EF - GenBank no. EF514914; Metastrongylus salmi - GenBank no. AY295809; Protostrongylus rufescens - GenBank no. AJ920364; Skrjabingylus chitwoodorum - GenBank no. AY295819; Stenurus minor - GenBank no. AY295817; Stroglostrongylus wilsoni - GenBank no. AY295820; Trichostrongylus colubriformis - GenBank no. AJ920350; A. costaricensis - GenBank no. GU587745; A. vasorum - GenBank no. GU045374.

\section{REFERENCES}

1. Spratt DM. Species of Angiostrongylus (Nematoda: Metastrongyloidea) in wildlife: a review. Int J Parasitol Parasites Wildl 2015; 4: 178-189.

2. Miyazaki I. Angiostrongyliasis. In Helminthic Zoonoses. Tokyo, Japan. International Medical Foundation of Japan. 1991, pp 347-355.

3. Rosen L, Chappell R, Laqueur GL, Wallace GD, Weinstein PP. Eosinophilic meningoencephalitis caused by a metastrongylid lung-worm of rats. JAMA 1962; 179: 620-624.

4. Phan TN. Angiostrongylus cantonensis. In Textbook for Training Medical Doctors of Hanoi University. Hanoi, Vietnam. National Hospital of Tropical Diseases. 2009, pp. 246-247 (in Vietnamese).

5. An PN. Some comments for eosinophilic meningoencephalitis in children in National Hospital of Children from 1995 to
2000. J Pract Med 2002; No. 3: 66-69 (in Vietnamese).

6. De NV. Report of eosinophilic meningoencephalitis caused by Angiostrongylus. J Pharm-Med Inform 2004; No. 5: 15-16 (in Vietnamese).

7. Hwang KP, Chen ER. Clinical studies on Angiostrongylus cantonensis among children in Taiwan. Southeast Asian J Trop Med Public Health 1991; 22(suppl): 194-199.

8. De NV, An PN, Huong TTT. Eosinophilic meningoencephalitis caused by Angiostrongylus. J Pharm-Med Inform 2008; No. 11: 9-13.

9. Xuan LT, Dung DH, Hien TV. A case infected by Angiostrongylus cantonensis in the eye in Ho Chi Minh City. J Med Ho Chi Minh City 2001; No. 1: 97-100 (in Vietnamese).

10. Feng Y, Nawa Y, Sawanyavisuth K, Lv Z, Wu ZD. Comprehensive review of ocular angiostrongyliasis with special reference to optic neuritis. Korean J Parasitol 2013; 51: 613-619.

11. Tamura K, Nei M, Kumar S. Prospects for inferring very large 
phylogenies by using the neighbor-joining method. Proc Natl Acad Sci USA 2004; 101: 11030-11035.

12. Qvarnstrom Y, da Silva ACA, Teem JL, Hollingsworth R, Bishop H, Graeff-Teixeira C, da Silva AJ. Improved molecular detection of Angiostrongylus cantonensis in mollusks and other environmental samples with a species-specific internal transcribed spacer 1-based TaqMan assay. Appl Environ Microbiol 2010; 76: 52875289.
13. Liu YJ, Han YP, Li ZY, Wei J, He HJ, Xu CZ, Zheng HQ, Zhan $\mathrm{XM}, \mathrm{Wu} \mathrm{ZD}, \mathrm{Lv} \mathrm{ZY}$. Molecular cloning and characterization of cystatin, a cysteine protease inhibitor, from Angiostrongylus cantonensis. Parasitol Res 2010; 107: 915-922.

14. Jefferies R, Shaw SE, Willesen J, Viney ME, Morgan ER. Elucidating the spread of the emerging canid nematode Angiostrongylus vasorum between Palearctic and Nearctic ecozones. Infect Genet Evol 2010; 10: 561-568. 
